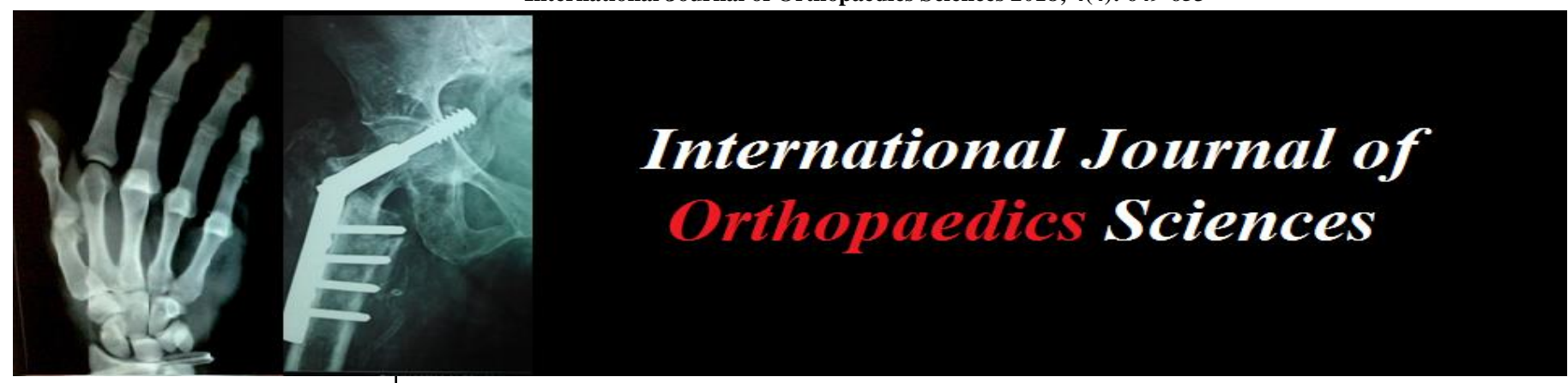

ISSN: $2395-1958$

IJOS 2018; 4(4): 649-653

(C) 2018 IJOS

www.orthopaper.com

Received: 19-08-2018

Accepted: 23-09-2018

Pavankumar Kohli

Professor Dept. of Orthopaedics,

BKL Walawalkar Medical

College, Dervan, Chiplun,

Ratnagiri, Maharashtra, India

\section{Ankush Nawale}

Fellow in spine surgery, Dept. of orthopaedics, BKL Walawalkar

Medical College, Dervan,

Chiplun, Ratnagiri,

Maharashtra, India

\section{Sushant Chavan}

Fellow in joint Replacement,

Dept. of Orthopaedics, BKL

Walawalkar Medical College,

Dervan, Chiplun, Ratnagiri,

Maharashtra, India

Satishchandra Gore

Mentor Spine Surgeon Dept. of Orthopaedics, BKL Walawalkar

Medical College, Dervan,

Chiplun, Ratnagiri,

Maharashtra, India

Sunil Nadkarni

Professor Dept. of Orthopaedics,

BKL Walawalkar Medical

College, Dervan, Chiplun,

Ratnagiri, Maharashtra, India

Correspondence

Ankush Nawale

Fellow in spine surgery, Dept. of orthopaedics, BKL Walawalkar

Medical College, Dervan

Chiplun, Ratnagiri,

Maharashtra, India

\section{Dervan dermatome syndrome often missed but surprisingly common coexistent spine pathology in 'Knee Pain', ignore at your own risk}

\author{
Pavankumar Kohli, Ankush Nawale, Sushant Chavan, Satishchandra \\ Gore and Sunil Nadkarni
}

DOI: https://doi.org/10.22271/ortho.2018.v4.i4h.78

\begin{abstract}
Introduction: Knee pain is among the commonest complaints in the outpatient orthopedic department. However the cause does not always originate from knee itself. and knee pain is used by the patient more as an umbrella term. In fact very few patients give the complete list of accompanying symptoms. They have more often than not to be coaxed out of the patient. To make matters more complex, Referred pain from the Spinal nerves, Hip and Ankle joints join the many structures around the knee to make knee pain an umbrella term for this Pandora's Box. Misdiagnosis or partial diagnosis leads to inadequate or wrong treatment and continued suffering for patient and treating doctors

Materials \& Methods: 78 patients with knee pain attended the OPD of Walawalkar hospital, Dervan. On x-ray, 28 were found to have different grades of osteoarthritis. Of these 25 agreed to be part of this pilot study. They underwent standing $\mathrm{x}$ rays \& MRI of the lumbosacral spine.

Results: This study found an unusually high rate of coexistent knee and spine pathologies. All patients had some degree of disc disease accompanying medial or tricompartmental osteoarthritis. It undermines the need for investigating this important contributor to the umbrella term of knee pain.

The commonest affected disc segment was L4-5 i.e 92\% with two patients having L5- S1 prolapse 8\%. The purpose of this pilot study is only to alert the surgical and rehabilitation colleagues of the frequent coexistence of the spine and knee conditions for Counseling, consent, surgery \& rehabilitation and thus improve patient satisfaction.
\end{abstract}

Keywords: knee pain, dermatome, ignorance, dervan

\section{Introduction}

Knee pain is among the commonest complaints in the outpatient orthopedic department. However the cause does not always originate from the knee itself ${ }^{[1,2]}$ and knee pain is used by the patient more as an umbrella term. In fact very few patients give the complete list of accompanying symptoms. They have more often than not to be coaxed out of the patient. To make matters more complex, Referred pain from the Spinal nerves, Hip and Ankle joints join the many structures around the knee to make knee pain an umbrella term for this Pandora's Box ${ }^{[3,4]}$. Misdiagnosis or partial diagnosis leads to inadequate or wrong treatment and continued suffering for the patient treating doctors ${ }^{[5,6]}$. We do now live in a litigious society.

This coexistence of spine and knee pathology also emphasizes need to

1. Investigate causality of knee pain and L4-5 pathology as chicken and egg syndrome.

2. Need to compulsively probe for coexistence of concomitant spine pathology with the umbrella term knee pain as it is the one factor which will influence complete diagnosis, counseling, legal consent taking, simultaneous treatment of both spine and knee disease and postoperative rehabilitation protocol.

3. Further Examine possibility of simultaneous treatment of knee pain and leg pain, i.e the entire Dermatome, by endoscopic discectomy and knee arthroplasty as a holistic and complete treatment and long term relief to the patient. 
There is a dire need, NOT to treat knee pain as a specialty in itself, thereby ignoring the holistic view of considering contributions to pain from the neighboring joints \& spine. Specialization should not lead to separation. Ignoring the role of the Spine as a pain generator ${ }^{[7,9]}$, is hazardous in a litigant society \& may lead to erroneous labeling of mildly arthritic $\mathrm{x}$ rays of the knee as the main pain causative agent.

\section{Materials and Methods}

The subjects were patients who visited Outpatient department for primary knee pain. This study has been conducted in BKL walawalkar rural medical college and research centre, Dervan, dist Ratnagiri, Maharashtra, India. The study was approved by Ethics committee. 78 patients visited the above hospital in the month of January 2018 with the primary complaint of knee pain. Of them 25 had confirmed Osteoarthritis on $\mathrm{X}$ rays. 25 of them consented to be part of this pilot study. Mean age of the patient was 60.4 years; there were 11 males and 14 females. All patients complained primarily of pain at knee joint. None of the patients complained of back pain or radiculopathy on their own.

We evaluated AP and Lateral view radiographs of Knee joint. Patients were further subjected to $X$ rays and MRI of Lumbosacral spine. Under a special research arrangement with the local MRI center, all patients were examined clinically for knee pain, general examination done to rule out any systemic neurological disorder. $\mathrm{X}$ rays took included Anteroposterior (AP) weight-bearing knee and standing Anteroposterior with Lateral radiographs of Lumbosacral spine and MRI of Lumbosacral spine axial and saggital view $\mathrm{T} 1$ and $\mathrm{T} 2$ images.

\section{Inclusion criteria}

1. Patient who consented for above mention investigation

2. Knee pain

3. Age group between 50 to 80 years

\section{Exclusion criteria}

1. Subjects with previous neurological disorder; Cerebro vascular attack, potts spine, charcots joints, previous surgery

2. Subject with Osteoarthritis secondary to trauma.

3. Generalized involvement RA, ankylosing spondylosis, Inflammatory / systemic disease.

Data was collected and analyzed by the Radiological imaging center and reported by a senior radiologist. Results were calibrated and presented in graphical form of pie charts.

\section{Results}

All patients showed varying degrees of disc disease. The commonest affected disc segment was L4-5 i.e 92\% with two patients having L5-S1 prolapse $8 \%$.

The work for further staging of the disc disease, its anatomical classification and its correlation with the grade of osteoarthritis is part of a larger study to be reported soon.

The purpose of this pilot study is only to alert the surgical and rehabilitation colleagues of the frequent coexistence of the spine and knee conditions for Counseling, consent, surgery \& rehabilitation.

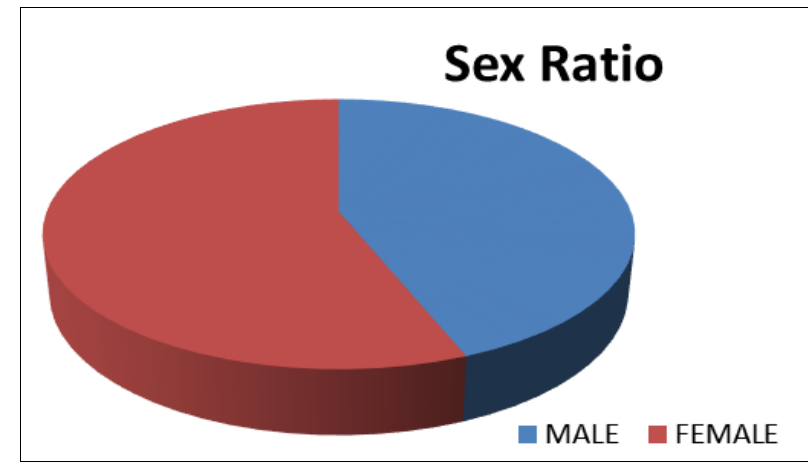

Chart 1

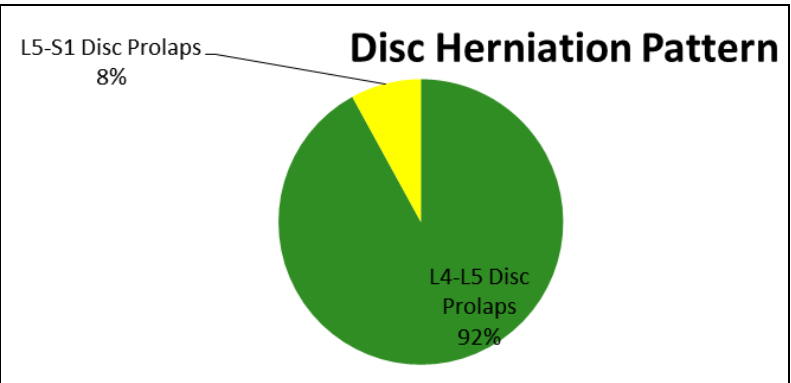

Chart 2

\section{Discussion}

The senior contributors of our team have studied the subject of knee pain with special reference to L5 radiculopathy $[1,2,10]$. Lateral knee pain has been shown to commonly arise from the L5 nerve root ${ }^{[1,2,10]}$.

Symptomatic effusion, patellofemoral pain, is common accompanying symptoms.

Also, there are many a well done arthroplasties who still complain of pain around the knee postoperatively [11]. We surmise that a predominant cause for the above is missing the element of coexistent spine pathology or not giving it due importance in counseling, rehabilitation and treatment. This sometimes needlessly leads to authentic doctors, well done surgeries \& a sound technique getting a bad name.

Majority of medial knee pains originate from the knee itself \& those lateral or posterolateral have a contribution of radiculopathy from the L5 nerve root ${ }^{[1,2]}$. All posterior or posterolateral pain complaints are questioned for past history of back pain and fresh, coexisting or previous radiculopathy symptoms. We have found an extremely high incidence of radiculopathy in the posterior \& posterolateral region of knee which correlates greatly with subsequent MRI of Lumbosacral spine. This is so far proven \& well accepted scientifically by our senior authors.

However, there was also a correlation to medial knee pain, often noted as L4 exiting root compression. The commonest culprit disc prolapse was L4-5.

It is too early for us to assert that the knee arthritis may be caused by commonly coexistent disc pathology of L4-5 disc prolapse. We further surmise that, could central disc herniations with traversing root L5 compression causing a posterior \& posterolateral knee pain/ calf pain cause abnormal load patterns due to weakness of hip abductors on that side ? Also could exiting or paracentral disc herniation may cause Medial sided or medial joint arthrosis cause exaggerated cartilage wear due to a subclinical hyposensitisation of the L4 sensory supply to medial side of knee? 
Further studies are needed to verify this theory.

Modifications in our treatment protocol are then made for Counseling, Rehabilitation and Treatment of such patients who may have coexistent spine and knee pathologies. We have developed "THE DERVAN PROTOCOL" for investigation which includes standing knee and spine X-rays and MRI spine.

Often on deep probing and explaining the concept of knee as a bulb, the nerve as a cable $\&$ Spine as a junction box, and that a fault in the bulb may be from the bulb or cable or junction box. The patient is able to relate to the pain generator concept and does remember an episode of back pain followed by radiculopathy etc.

The patient who has knee osteoarthritis which may need surgery is then counseled for the causative contributors to the pain. If required symptomatically \& proved by imaging,

Patient is then offered either Knee surgery with special focus on concomitant rehabilitation of spine, a training of day to day correct postural ergonomics. If both knee arthritis \& spinal canal stenosis are equally severe, we offer him concomitant surgery by the "Dervan Simultaneous Surgery Protocol "which is also under review for publication. We are also happy to state that we are the first team to treat simultaneous knee and spine pathologies, in the same sitting. i.e endoscopic decompression of spine followed by endoscopic insertion of epidural catheter \& simultaneous surgery of the knees in an awake \& aware patient. This leads to complete readdressal of all causative pain factors concomitantly. However this paper is to report the imperative need for the diagnosis rather than treatment. We have called it the "Dervan Dermatome Syndrome"

Rehabilitation of the patient is done following the "Dervan Rehabilitation Protocol" which is also under review for publication. To our knowledge this is the first study to report the high incidence of knee and spine pathologies and its ramifications.

Knee pain is commonly considered an independent and separate clinical entity in itself. Specialization has unfortunately led to separation. A knee pain specialist is concerned more with the MRI and X rays of the knee and seldom seeks to find the alternate or maybe even true pain generator after a positive imaging finding in the knee. Unfortunately even after proper and complete readdressal of the image by knee surgery, many patients complain of persistent pain, to be slowly referred to other colleagues including counselors. Practicing as a holistic team comprising arthroplasty and spine experts along with pain medicine specialists, at the Rural Medical College in Dervan gave us the opportunity to probe for all possible causes of knee pain, confirm by radiology imaging and then redress all of them together.

This enumeration of all causes of pain gave us a much better and complete approach to counseling preoperatively, surgical readdressal either concomitantly or in planned stages and focusing simultaneously on all needy areas during rehabilitation.

\section{Example Case}

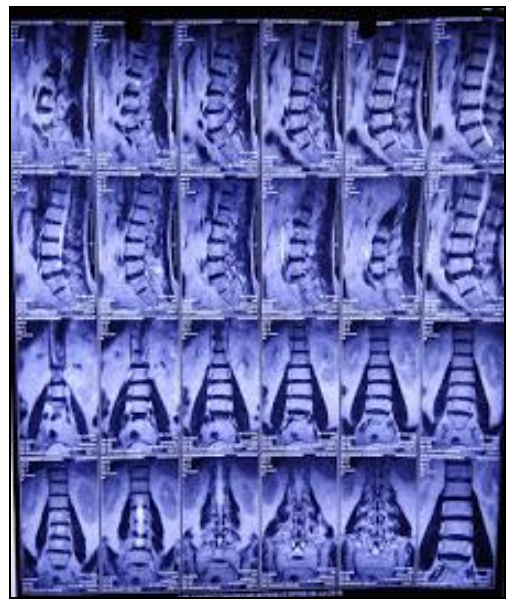

Image 1: MRI Lumbosacral Spine

Diffuse annular disc bulge along with bilateral facetal ligamental hypertrophy at L4-5 Levels Causing Moderate significant impression on thecal sac and bilateral lateral recess nerve roots and compromising bilateral neural foramina. Patient complained of pain over lateral \& medial knee. On Questioning, He also confessed to calf pain \& repeated history of radiculopathy over past 2 years. But he insisted that the pain was presently only Knee pain!
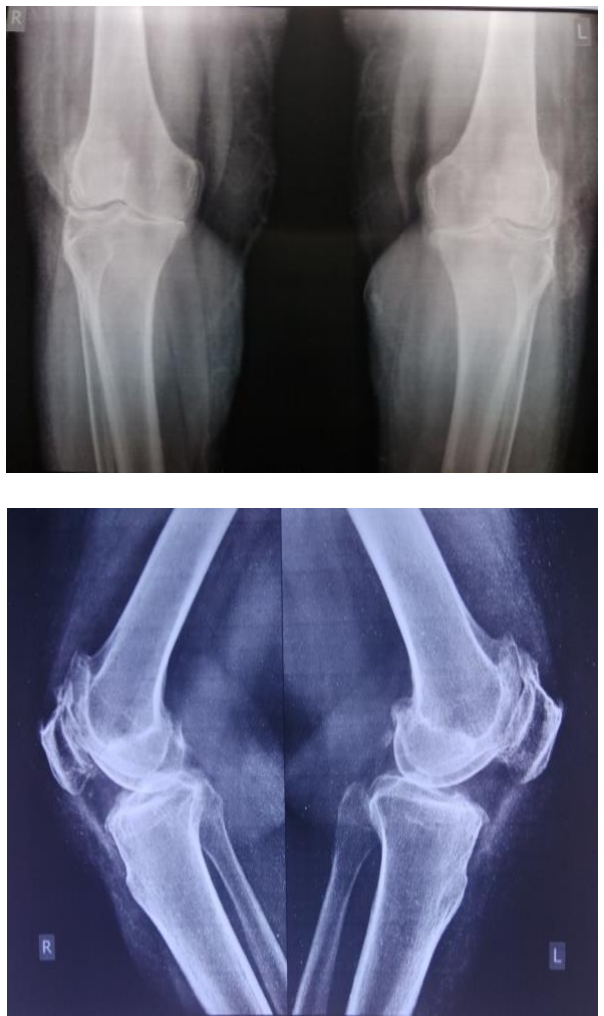

Image 2, 3: Bilatoral Knee Xray Anteroposterior and Lateral View Showing Degenerative Osteoarthritis with Cartilage Thinning 


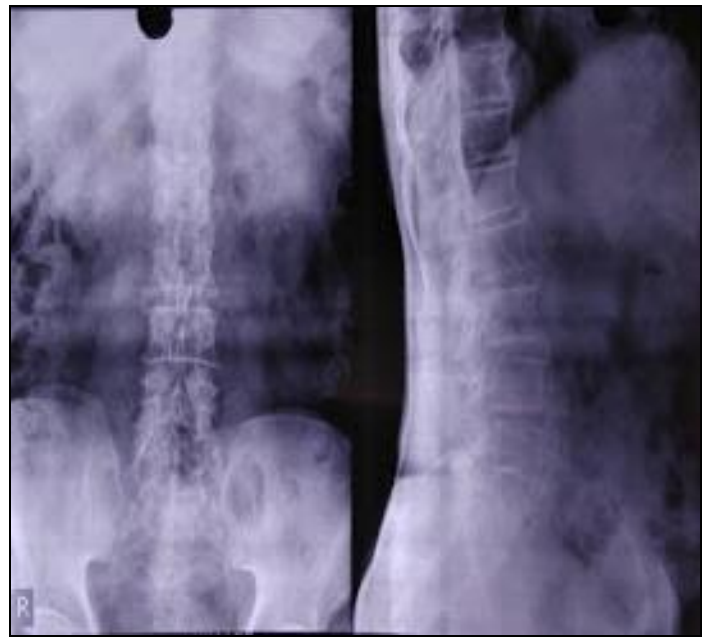

Image 4, 5: Xray Lumbosacral Spine Anteroposterior and Lateral view showing reduced disc space

\section{Conclusion}

The epidemiology of patients who present with radicular complaints and symptoms of knee osteoarthritis often overlaps and can prove difficult to correctly diagnose. Additionally, pre-existing arthritis of an extremity can cause imbalance in the spine, leading to mal alignment and radicular symptoms. One must be aware of the multiple causes of hip, knee, and back pain and how to properly examine these patients.

A new method for Work up for these complaints to make the correct diagnosis and guide treatment is proposed. Special vigilance should be paid to postoperative patients with new back pain several years after arthroplasty. With rising super specialization, a team approach to patients with potentially atypical presentations of their joint or back pain is best for optimizing the appropriate care.

In our study all patients with knee pain\& osteoarthritis on $\mathrm{x}$ rays who underwent set of investigation as described above have some pathology at L4-5 disc level. This concludes that there is need for considering knee pain and back pain together and investigation as a single functional unit \& treat accordingly with relevant set of investigations. We can presently treating symptomatic knee arthritis and symptomatic prolapse inter vertebral disc in a single surgical setting. This leads to better and early Rehabilitation of patient and will prevents dissatisfaction because of persistence of the umbrella term "pain around the knee". Hence we suggest following the new investigation protocol in all patients with knee pain along with back pain for holistic healing.

Also further avenues of research are opened by this paper on the causation of knee pain, is the affliction of the spine $\&$ knee a chicken \& egg syndrome, if so, which precedes which? Are there ways to quantify the contribution to knee pain, thus prioritizing the sequence of surgical intervention?

Can specific rehabilitation protocols be developed for such patients who focus on back care too along with knee rehabilitation, as a more holistic approach?

Can we also further develop minimally invasive techniques to address the entire dermatome simultaneously, in a single sitting, thereby giving complete relief to the patient?

The future looks exciting \& we are also reminded of the wisdom of our ancient wise ones who called the spine "MERUDANDA or the AXIS OF THE UNIVERSE", where every man is a universe in himself $\&$ the axis is the human spine.

\section{Acknowledgement}

We are grateful to Dr Ravishankar Vedantam, Spine Surgeon, and USA for his guidance in preparing this manuscript.

\section{References}

1. Sciatica detection and confirmation by new method. Dr Satishchandra Gore, Dr. Sunil Nadkarni, International journal of Spine Surgery. 2014; 8:15.

2. Stable Devlopmental Lumbar Canal Stenosis, Rethink needed Editorial JAOS, 2018.

3. Andersen RE, Crespo CJ, Bartlett SJ, Bathon JM, Fontaine KR. Relationship between Body Weight Gain and Significant Knee, Hip, and Back Pain in Older Americans. Obesity Res. 2003; 11:1159-62

4. Felson DT, Zhang Y. An update on the epidemiology of knee and hip osteoarthritis with a view to prevention. Arthritis Rheum. 1998; 41:1343-55.

5. Dr Allan. A van Zyl Misdiagnosis of hip pain could lead to unnecessary spinal surgery. SA orthop. J Centurion Jan. 2010; 9:4

6. Massimo Allegri, Silvana Montella, et al. Mechanisms of low back pain: a guide for diagnosis and therapy Version 1. F1000Res. 2016; 5:F1000. Faculty Rev-1530. Published online 2016 Jun 28 PMCID: PMC4926733

7. Mohammad Rahbar, et al. Association between knee pain and low back pain.J Pak Med Assoc. 2015; 65(6):626-31

8. Masahiko Ikeuchi, Masashi Izumi, Koji Aso, Natsuki Sugimura, and Toshikazu Tani. Clinical characteristics of pain originating from intra-articular structures of the knee joint in patients with medial knee osteoarthritis Springerplus. 2013; 2:628. Published online 2013 Nov 23. doi: [10.1186/2193-1801-2-628.

9. Clinton J Devin, Kirk A Mccullough et al. Hip-spine Syndrome The Journal of the American Academy of Orthopaedic Surgeons. 2012 20(7):434-42.

10. Dr. Satishchandra Gore, Ms Smruti Gore. Identifying lateral knee pain using sodium channel blockers at Ankle, International Journal of Engineering Science Invention IJESI, 2016, 1-3

11. Hong-An Lim, MD, Eun-Kyoo Song, MD, et al. Causes of Aseptic Persistent Pain after Total Knee Arthroplasty. Clin Orthop Surg. 2017; 9(1):50-56. Published online. 2017 Feb 13. doi: [10.4055/cios.2017.9.1.50]

12. DeFroda F, MDA Alan, Daniels H, et al. Differentiating Radiculopathy from Lower Extremity Arthropathy the American Journal of Medicine. 2016; 129:10.

13. Rubino FA ${ }^{1}$ Gait disorders. Neurologist. 2002; 8(4):25462.

14. Yun Peng Huang, ${ }^{2}$ Sjoerd M. Bruijn Gait adaptations in low back pain patients with lumbar disc herniation: trunk coordination and arm swing Eur Spine J. 2011; 20(3):491-499 [PubMed]

15. Morag $\mathrm{E}^{1}$, Hurwitz DE Abnormalities in muscle function during gait in relation to the level of lumbar disc herniation. Spine (Phila Pa 1976). 2000; 25(7):829-33. [PubMed]

16. Shengzheng Kuai, Zhenhua Liao et al. The Effect of Lumbar Disc Herniation on Musculoskeletal Loadings in the Spinal Region During Level Walking and Stair Climbing. Med Sci Monit. 2017; 23:3869-3877. [PubMed]

17. Shengzheng Kuai, Wenyu Zhou. Influences of lumbar disc herniation on the kinematics in multi-segmental spine, pelvis, and lower extremities during five activities of daily living, 2016. 
18. MC Musculoskeletal Disorders BMC series. https://doi. org/10.1186/s12891-017-1572-7

19. Wolfe F, Hawley D, Peloso P, Wilson K, Anderson J. Back pain in osteoarthritis of the knee. Arthritis Care Res. 1996; 9:376-83.

20. Wolfe F. Determinants of WOMAC function, pain and stiffness scores: evidence for the role of low back pain, symptom counts, fatigue and depression in osteoarthritis, RA and fibromyalgia. Rheumatology. 1999; 38:355-61.

21. Pozzi F, Snyder-Mackler L, Zeni J. Physical Exercise After Knee Arthroplasty: A Systematic Review Of Controlled Trials Eur J Phys Rehabil Med. 2013; 49(6):877-892.

22. Jaydev B. Mistry, Randa DK. Elmallah. Rehabilitative Guidelines after Total Knee Arthroplasty: A Review. The Journal of Knee Surgery. DOI: 10.1055/s-0036-1579670. 